\title{
LAS PRECIPITACIONES DE LA ÉPOCA CÁLIDA EN EL SUR DE LA PROVINCIA DE ALICANTE DESDE 1550 A 1915
}

\author{
José Creus NOVAU \\ Instituto Pirenaico de Ecología-CSIC \\ Miguel A. SAZ SÁNCHEZ \\ Departamento de Geografia-Universidad de Zaragoza
}

\section{Resumen}

Se analizan las principales anomalías de la precipitación de primavera y verano en el sur de la provincia de Alicante, reconstruidas mediante técnicas dendroclimáticas. Su análisis permite destacar una alternancia de periodos secos (1598-1651, 1671-1687 y 1764-1781) y húmedos (1650-1670 y 1688-1739) de desigual duración e intensidad. A partir del siglo XIX las anomalías de uno y otro sentido son más cortas, aunque su intensidad es similar.

Palabras clave: Dendroclimatología, anomalías de la precipitación, siglos XVI-XIX, Pequeña Edad del Hielo, Alicante, España.

\begin{abstract}
In this paper we analyze the main anomalies of spring and summer precipitation in the south of the province of Alicante (SE of Spain), reconstructed using dendroclimatological techniques. We have identified an alternation of dry periods (1598-1651, 1671-1687 and 1764-1781) and humid (1650-1670 and 1688-1739) of different duration and intensity. In the 19 th century the rainfall anomalies are shorter, although of similar intensity.

Keywords: Dendroclimatology, rainfall anomalies, 16th-19th centuries, Little Ice Age, Alicante, Spain.

\section{1.- INTRODUCCIÓN}

Durante la década de los años setenta tuvieron lugar, a escala mundial, una serie de acontecimientos climáticos adversos (sequía en el Sahel, monzones debilitados, nieve a nivel del mar a $25^{\circ} \mathrm{LN}$, etc) que trajeron a un primer plano de la actualidad el concepto de normalidad del clima. Las consecuencias económicas y sociales de tales
\end{abstract}


episodios fueron muy importantes, hasta el punto de evidenciar que cualquier desviación del comportamiento «normal» del clima tiene un gran impacto sobre los sectores productivos, aunque estén bien adaptados a su medio.

El clima de la tierra ha sido y sigue siendo, por propia naturaleza, cambiante a lo largo del tiempo y pueden citarse muchos ejemplos de cómo determinados acontecimientos meteorológicos, incluso oscilaciones y pulsaciones climáticas, han tenido importantes consecuencias sociales y políticas sobre determinados países o regiones'. Especialmente en épocas pasadas cuando el bienestar de las comunidades humanas tenía una dependencia muy directa de las condiciones atmosféricas, pero también en la actualidad cuyas actividades agrarias continúan en gran medida supeditadas a la bondad del clima².

No es de extrañar que, en determinados momentos, la escasez o falta de alimentos fueran causa de inestabilidad y conflictividad social, capaz incluso de provocar migraciones en aquellas zonas donde la persistencia de determinados comportamientos anómalos del clima afectaban de forma continuada las economías agrarias de subsistencia.

Por ello, el estudio del clima pasado puede ser de gran interés para comprender y explicar determinados acontecimientos, especialmente los referidos a aquellos siglos pasados en los que la actividad económica tenía una total dependencia del clima y sus anormalidades, aunque fueran puntuales, producian un fuerte impacto social y políti$\mathrm{Co}^{3}$. Como indican algunos autores ${ }^{4}$, la posibilidad de que los cambios climáticos puedan jugar un papel en la explicación histórica constituye una de las dimensiones más excitantes del estudio del clima pasado.

Su análisis no plantea problemas a partir del $\mathrm{s}$. XX, y de forma excepcional desde finales del s. XIX cuando se inicia la toma de datos con instrumental adecuado. Sin embargo, no resulta tan sencillo estudiarlo en épocas anteriores carentes de datos cuantificados. En este caso hay que recurrir a los proxy-data que aportan distintas fuentes de información indirectãs englobadas dentro de la paleoclimatología. Para este trabajo se ha utilizado la dendroclimatología, es decir, la climatología reconstruida a partir del crecimiento anual de los árboles. Se trata de una información cuantificada y objetiva, de total continuidad temporal y con una resolución anual e incluso trimestral.

La finalidad de este trabajo es aportar información sobre el clima del sur de la provincia de Alicante desde mediados del siglo XVI hasta principios del siglo XX, a partir de la reconstrucción de la precipitación del observatorio de Elche mediante dicha técnica. En concreto se han reconstruido las lluvias del periodo cálido (abril a septiembre), a su vez desglosadas entre las de primavera (abril-mayo-junio) y verano (julio-agosto septiembre). Ambos periodos son los de menor precipitación intranual, reflejado en las

1. Olcina, J. y MARTin, J.: La influencia del clima en la historia, Madrid, 1999, 96 págs.

2. ZAMORA, R: El final de la Pequeña Edad del Hielo en Alicante, Alicante, 2002, 194 págs.

3. BAUERNFEIND, W. y WOITEK, U.: «The influence of climatic change on price fluctuations in Germany during the 16th century Price Revolution». Climatic Change, vol. 43, 1999, págs 303-321.

4. DE VRIES, J.: «Measuring the impact of climate on history: The search for appropiate methodologies». Climate and History. Londres, 1981, págs 19-50. 
prolongadas sequías que afectan a toda esta zona en la actualidad y que en siglos pasados pudieron tener gran trascendencia en el acontecer diario de sus gentes.

\section{2.- LA FUENTE DE INFORMACIÓN DENDROCRONOLÓGICA}

Durante los años noventa, y en el marco de varios proyectos de investigación, se realizaron numerosos muestreos en gran parte del territorio peninsular en busca de poblaciones de árboles viejos donde elegir ejemplares varias veces centenarios. El banco de muestras conseguido supera las 1500 y con ellas se han construido más de 50 dendrocronologías con las que se han realizado reconstrucciones de variables climáticas en algunas zonas de España publicadas en diferentes trabajos ${ }^{5}$.

Siete de estas cronologías (mapa 1), situadas en el sureste peninsular, se han utilizado para reconstruir la precipitación de la época cálida en el observatorio de Elche (Alicante) y conocer su evolución plurisecular desde mediados del siglo XVI. En las localidades indicadas se muestrearon bosques de Pinus nigra Arnold. subsp. salmannii situados entre 1500 y $1850 \mathrm{mts}$ de altura, donde todavía es posible encontrar algunas poblaciones casi relictas de esta especie, preservadas por la altitud y dificultad de acceso. En cada lugar se buscaron los ejemplares de aspecto más vetusto, a los que se extrajo dos muestras radiales de su secuencia de anillos de crecimiento por medio de una barrena Pressler. Después de efectuar su medición (medidor Aniol con precisión de $0,01 \mathrm{~mm}$ ) se sincronizaron y estandarizaron según la metodología tradicional ${ }^{6}$ En la tabla 1 figuran las características de las series de índices estándar de las siete cronolo-

5. Entre otros podemos señalar los publicados por: Creus, J. y PuIgdefábregAs, J.: «Climatología histórica y dendrocronología de Pinus nigra Arnold». Avances sobre la Investigación en Bioclimatología, Madrid, 1983, págs 121-127; GénOva, M.: Dendroecología de Pinus nigra Arnold subsp. salzmannii (D) Franco y Pinus sylvestris L. en el Sistema Central y en la Serranía de Cuenca. Tesis Doctoral, Madrid, 1994, 460 págs; Creus, J., FERNÁNDEZ, A. y MANRIQUE E.: «Evolución de la temperatura y precipitación anuales desde el año 1400 en el sector central de la depresión del Ebro». Lucas Mallada, vol 8, Huesca, 1996, págs. 9-27; FernÁndez, A., GÉNOVA, M., CREus, J. y Gutiérrez, E.: «Dendroclimatological investigation for the last 300-400 years in Central Spain». Radiocarbon, 1996, págs 181-190; Creus, J. y SAZSÁNCHEZ, M. A.: «Estudio de la variabilidad climática del último milenio a partir de series de temperatura y precipitación reconstruidas en el noreste español». La Climatología española en los albores del siglo XXI. Barcelona, 1999, págs 155-164; SAZ-SÁNCHEZ, M. A. y CREUS, J.: «Comportamiento cíclico de la precipitación estival en el Noreste de España», A Geofisica e a Geodesia no século XXI, Lisboa, 2000, págs 515-516; CANDELA, V. (2000): Reconstrucción dendroclimática de las zonas Central y Meridional de España. Tesis Doctoral, Madrid, 2000, págs 497; SAZ-SÁNCHEZ, M.A. (2003): Temperaturas y precipitaciones en la mitad norte de España desde el siglo XV. Estudio dendroclimático. Zaragoza, 2003, págs 294; SAZ-SÁnChez, M. A., CReus, J. y CuADrAT, J. M.: «Mean summer temperatures dendroclimatic reconstruction in Northeast of Spain. Comparison with other regional studies». Geophysical Research Abstracts, vol 5, Niza, 2003, CD-rom; SAZ-SÁNCHEZ, M. A. y CREUS, J.: «Thermische anomalien im nördlichen Spanien während des 16. Jahrhunderts: erste zeichen der kleinen eiszeit». Landnutzungswandel und Landdegradation in Spanien. Frankfurt, 2003, págs 121-141.

6. FRITTS, H.: Tree rings and climate. Londres, 1976, págs 567; COOK, E.R. y KAIRIUKSTIS L. A.: Methods of dendrochronology. Londres, 1990, págs 394; FRITTs, H.: Reconstructing large-scale climatic patterns from tree-ring data. Tucson, 1991, págs 420. 


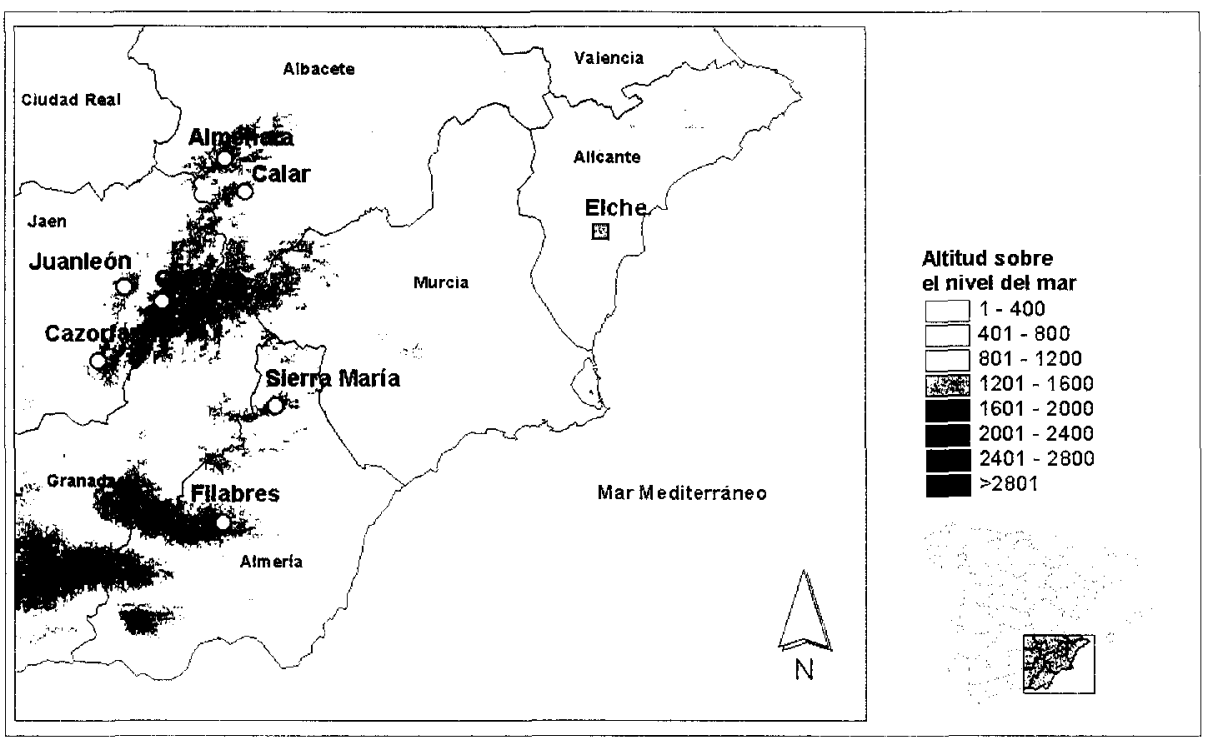

Mapa 1.- Situación de las dendrocronologías (círculos blancos) calibradas con el observatorio meteorológico de Elche.

gías. De ella se comentarán aquellos estadísticos que mejor indican su aptitud para ser usadas en reconstrucciones de clima. Tal es el valor de sensibilidad media (SM), que muestra la variabilidad interanual de los crecimientos radiales y, por tanto, una forma de cuantificar la sensibilidad de los árboles al clima. La tasa señal/ruido (S/R) es un indicador de la cantidad de señal climática de la serie media y refleja su capacidad para estimar la variabilidad general de la población muestreada. Valores superiores a 4,5 se consideran suficientes, superado en todas las cronologías salvo la de Sierra María donde no se alcanza por muy poco. Finalmente está la concordancia poblacional (CP), que muestra el grado de representatividad de la cronología respecto de la hipotética cronología que represente el conjunto de la población. En todos los casos se supera el valor $0,75-0,80$, considerado suficiente al asumirse una incertidumbre en torno al $20 \%$.

Globalmente, todos los indicadores alcanzan y superan los umbrales recomendados, salvo las cronologías de Galapán y Calar donde algunos quedan ligeramente por debajo del valor teórico. Probablemente esté relacionado con el menor número de muestras incluidas en ambas cronologías, debido a la necesidad de eliminar algunas de ellas por presentar discontinuidades o anomalías en el crecimiento. Dado que dichos valores son próximos a los umbrales requeridos, las hemos considerado válidas para ser utilizadas en reconstrucciones del clima. 


\begin{tabular}{|l|l|l|l|c|c|c|c|c|c|c|}
\hline Localidad & Provincia & Altitud & Especie & Años & $\mathbf{N}^{\circ} \mathbf{M}$ & SM & DE & Lag.1 & S/R & CP \\
\hline S. María & Almería & $1850 \mathrm{~m}$ & P. nigra A & 393 & 31 & 0,19 & 0,23 & 0,43 & 4,8 & 0,82 \\
\hline Filabres & Almería & $1800 \mathrm{~m}$ & P. nigra A & 337 & 12 & 0,24 & 0,30 & 0,52 & 5,6 & 0,88 \\
\hline Juanleón & Jaén & $1600 \mathrm{~m}$ & P. nigra A & 385 & 25 & 0,28 & 0,21 & 0,47 & 5,8 & 0,85 \\
\hline Galapán & Jaén & $1550 \mathrm{~m}$ & P. nigra A & 317 & 11 & 0,19 & 0,21 & 0,46 & 5,0 & 0,78 \\
\hline Cazorla & Jaén & $1850 \mathrm{~m}$ & P. nigra A & 939 & 20 & 0,22 & 0,21 & 0,48 & 9,7 & 0,90 \\
\hline Calar & Albacete & $1490 \mathrm{~m}$ & P. nigra A & 336 & 19 & 0,19 & 0,23 & 0,47 & 6,2 & 0,76 \\
\hline Almenara & Albacete & $1650 \mathrm{~m}$ & P. nigra A & 409 & 19 & 0,20 & 0,29 & 0,58 & 5,8 & 0,85 \\
\hline $\begin{array}{l}\text { No M = Número de muestras; SM = Sensibilidad Media; DE = Desviación Estándar; Lag.1 = Autocorrelación } \\
\text { de primer orden; S/R = tasa Señal/Ruido; CP = Concordancia poblacional. }\end{array}$ \\
\hline
\end{tabular}

Tabla 1.- Características de las cronologías estándar utilizadas.

\section{3.--RECONSTRUCCIÓN DEL CLIMA}

La metodología tradicionalmente empleada se basa en la utilización de un número de cronologías más o menos próximas al observatorio meteorológico que se quiere reconstruir ${ }^{7}$. Los valores climáticos, objeto de reconstrucción, actúan como variables independientes que deben explicar la variabilidad del crecimiento de los árboles bajo una dependencia del clima. Por medio de regresiones en componentes principales se calcula este sistema de relaciones (función respuesta), que indican y cuantifican en qué medida el clima condiciona el crecimiento y qué variables climáticas son las más importantes en este proceso y por tanto susceptibles de ser reconstruidas. Posteriormente este proceso se hace reversible y las variables dependientes (incrementos anuales) pasan a ser predictores de los valores climáticos que más influyen en dicho crecimiento, tantos años hacia el pasado como años tengan las cronologías.

Modificaciones posteriores de dicha metodología ${ }^{8}$ han permitido progresar en este sentido al posibilitar la utilización de un mayor número de cronologías, aunque estén distantes. Con ello se consigue que las reconstrucciones del clima sean más consistentes en el tiempo y posibles en más lugares. Tal propuesta se basa en la existencia de relaciones estadísticas entre elementos del clima registrados en observatorios situados a distancias importantes ${ }^{9}$ y que quedan recogidas en las dendrocronologías, como lo demuestra el proceso de sincronización existente entre árboles distantes entre sí más

7. FRITTS, H.: «Modelling tree-ring and environmental relationships for dendrochronological analisys». Forest growith process modelling of responses to environmental stress, Oregon, 1990, págs 360-382; Guior, J. (1990): «Methods of calibration». Methods of dendrochronology. Londres, 1990, págs 165-172.

8. FERNANDEZ, A. y MANRIQUE, E.: Nueva metodologia para la reconstrucción dendroclimática y aplicaciones más importantes, Madrid, 1998, págs 127.

9. VilLL, D., Guerra, I. y Corres, R.: Análisis estadístico de la pluviometria de la península lhérica. Madrid, 1985, págs 37. 
de $350 \mathrm{~km}^{10}$. De esta forma, al poder utilizar más cronologías es posible reconstruir un mayor número de variables climáticas.

Las muestras que intervienen en el proceso de reconstrucción se seleccionan en función de su correlación con las variables climáticas y, según la zona de procedencia, se configuran regiones dendrocronológicas basadas en criterios de coherencia geográfica y uniformidad climáticas. Las muestras elegidas en cada una de esas regiones se sincronizan y se estandarizan para conseguir una cronología estándar de la región.

A partir de ese momento, el proceso sigue la metodología tradicional, calculando regresiones en componentes principales entre las series de crecimientos que configuran las distintas regiones dendrocronológicas y las variables climáticas del observatorio elegido, consideradas para el año en curso y también anterior dada su persistencia sobre el crecimiento. La fiabilidad del resultado viene expresado por los coeficientes de los componentes obtenidos en el proceso de calibración-verificación aplicado a distintos intervalos mediante técnicas bootstrap hasta conseguir estabilizar la varianza y calculando el número de series de anillos que por azar tendrían correlación significativa con las variables climáticas. Esta nueva variable se compara con algunas distribuciones (normal, weibull, gamma) para conocer si las reconstrucciones son distintas de un simple proceso aleatorio. Por consiguiente, una variable climática reconstruida se considera significativa si supera los procesos de calibración, verificación y simulación.

\section{4.- RESULTADOS}

La elección del observatorio de Elche (Alicante) para ser reconstruido en el sureste español fue debido a que cumple dos requisitos indispensables para llevar a cabo el proceso metodológico: tener una serie de datos sin lagunas y una longitud de registro superior a 45 años, imprescindibles para obtener resultados con significación estadística. Aunque pueda parecer extraño, son pocos los observatorios de esta zona, y de muchas otras de nuestro país, que cumplen tales requisitos. Por ello, a menudo la elección del lugar deja pocas alternativas.

Aplicando el proceso metodológico antes descrito y calibrando los índices de crecimiento con los valores climáticos del observatorio de Elche fue posible reconstruir la precipitación de primavera (abril-mayo-junio) y verano (julio-agosto-septiembre) desde 1550 a 1915, además de la del periodo cálido (abril a septiembre) sumando los valores de ambas épocas.

\begin{tabular}{|l|c|}
\hline & $\mathbf{R}^{2}$ \\
\hline Precipitación de primavera & $0,77(\alpha=0.01)$ \\
\hline Precipitación de verano & $0,64(\alpha=0.01)$ \\
\hline
\end{tabular}

Tabla 2.- Varianza del crecimiento explicada por el clima.

10. GÉnOVA, R.: «Sincronización entre distintas cronologías del noreste de la Península Ibérica». Options mediterraneénnes, vol 3, 1988, págs 337-340. 
La tabla 2 recoge los valores que expresan la relación entre los datos utilizados en la calibración y los obtenidos con el modelo calculado. Aunque difieren ligeramente entre una y otra variable, superan ampliamente el umbral estadístico exigido para una significación del 99\%.

\subsection{Variabilidad de la precipitación semestral y trimestral}

Una primera aproximación al comportamiento secular de la precipitación reconstruida para el periodo cálido podemos hacerla analizando los coeficientes de variación móviles (CV) calculados con una amplitud de 25 años y representados en la figura 1.

En ella destaca la gran variabilidad de la precipitación hasta mediados del siglo XVIII, especialmente la de verano cuyo valor medio del $\mathrm{CV}$ es próximo al $60 \%$, para luego mantenerse de forma sostenida hasta finales del siglo XVII. A su vez, la lluvia de la primavera también presenta valores altos del CV aunque en menor medida (valor medio en torno al $40 \%$ ), debido a la alternancia de fases muy variables similares al verano (primer cuarto del siglo XVII) con otras mucho más estables (tercer cuarto del siglo XVII).

Después de unos decenios de precipitaciones más estables a mediados del siglo XVIII, la variabilidad se incrementa de forma progresiva hasta configurar otro periodo caracterizado por lluvias muy irregulares. Dicho incremento de la variabilidad pluviométrica sólo persiste hasta el primer cuarto del siglo XIX respecto al verano, mientras que la irregularidad primaveral perdura hasta el segundo cuarto y alcanza un máximo entre los años 1825-1850.

Desde entonces la irregularidad de ambas épocas disminuye de forma continuada, manteniendo esa tendencia la precipitación de primavera, mientras que la del verano tiende a incrementarse ligeramente hasta comienzos del siglo XX en que finaliza la

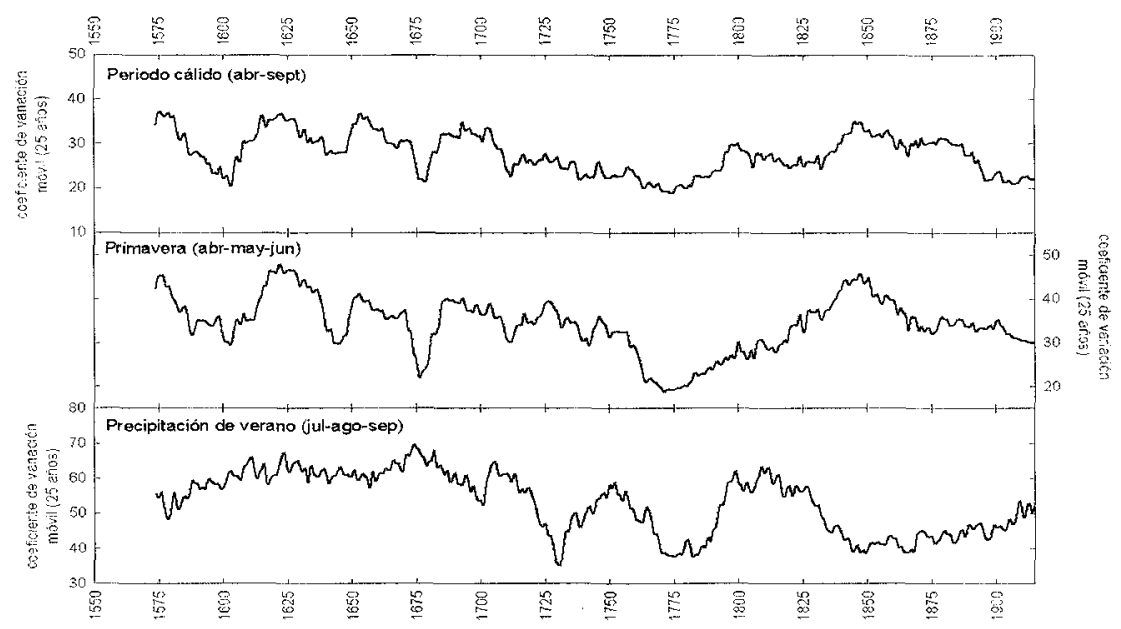

Figura 1.-Valores del coeficiente de variación móvil (\%). El valor asignado a un año corresponde al coeficiente de variación de los 25 anteriores. 
serie reconstruida, lo cual indica un mayor peso de la precipitación de primavera en la configuración del régimen de lluvias de abril a septiembre.

\section{2.- Análisis climático de la precipitación.}

Los valores de precipitación (periodo cálido, primavera y verano) se han representado en la figura 2 en forma de anomalías estandarizadas respecto a la media de todo el periodo 1550-1915. Para su mejor visualización se han suavizado mediante filtro gaussiano de corta amplitud (10 años) para resaltar los periodos lluviosos o secos, sin que ello impida observar y conocer el valor de algún año concreto. A su vez, en la tabla 3 se han reunido los cinco periodos más anómalos (secos o lluviosos) considerados en intervalos de 10, 5 y 3 años.

En este punto conviene recordar que los porcentajes de aumento o disminución de la precipitación que se indicarán para alguno de estos periodos incluyen años con valores muy distintos a los que caracterizan este intervalo. Por este motivo, una determinada anomalía seca podría cuantificarse por un mayor porcentaje de disminución de la precipitación si se recortara su duración y quedaran fuera años normales o lluviosos. $Y$ viceversa con las anomalías húmedas. Tal posibilidad nos llevaría a analizar los datos en grupos de 2 ó 3 años, etc, y delimitar periodos anómalos mucho más pronunciados y quizá más coincidentes con las noticias escritas que hacen referencia a lo acontecido en un año concreto o parte de un año, pero ello nos alejaría del objetivo del trabajo cual es mostrar las principales fases anómalas. Cuando la necesidad o curiosidad sea aquella, en la figura 2 pueden verse los datos año a año (línea discontinua) y el lector puede hacer las agrupaciones que más le interese conocer en un determinado momento.

Según lo expuesto y aplicando un filtro de 10 años, a lo largo de estos 366 años reconstruidos se observan tres fases en las que la pluviometría del periodo cálido es especialmente reducida en esta zona del SE español: la primera mitad del siglo XVII, las décadas de los años setenta y ochenta de esa misma centuria y las décadas de los sesenta y setenta del siglo XVIII. Entre ellas se intercalan dos periodos más lluviosos correspondientes a los años 1650-1670 y 1688 y 1739 . Desde finales del siglo XVIII y hasta principios del siglo XX alternan intervalos secos y húmedos de duración más reducida, sin que se produzcan anomalías tan prolongadas como las identificadas en los siglos XVII y XVIII. En los apartados siguientes se comentan cada una de ellas.

\subsection{1.- La disminución de la precipitación en la primera mitad del siglo XVII}

Durante la primera mitad de este siglo tiene lugar la más prolongada de las crisis secas observadas en los valores reconstruidos. En concreto, entre 1598 y 1651 la precipitación total del periodo cálido queda un $18 \%$ por debajo de la media de los siglos analizados, y a pesar de la breve recuperación que se produce entre los años 1618 y 1622. Sólo el 25\% de los años superan los $84,8 \mathrm{~mm}$ de media del periodo cálido. En esta primera mitad del XVII se localizan buena parte de los decenios, quinquenios y trienios más secos del periodo analizado, así como cuatro de los cinco periodos cálidos con menor precipitación (tabla 3). Como ejemplos cabe destacar el decenio 1601-1610 


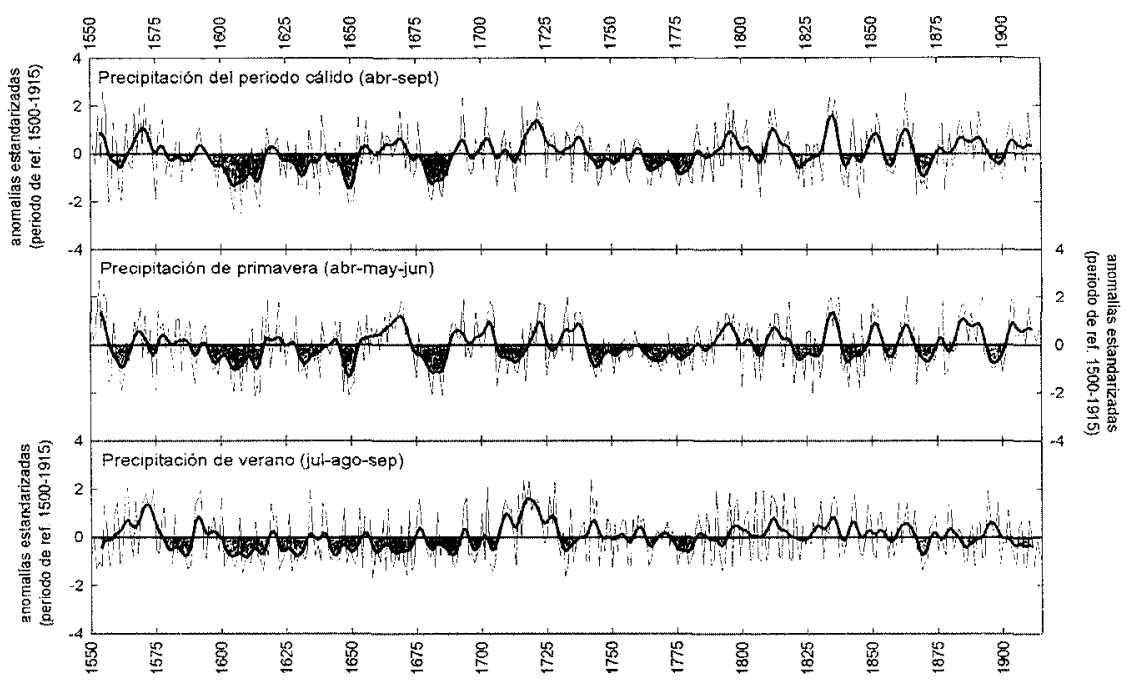

Figura 2.-- Valores de precipitación durante la época cálida (primavera + verano), durante la primavera y durante el verano en la zona de Elche entre 1550-1915, expresados en forma de anomalías respecto a la media de dicho periodo. Suavizado con filtro gaussiano de 10 años.

en que la precipitación se redujo un 33\% respecto de la media y que disminuye hasta el $50-60 \%$ en algún intervalo de 5 y 3 años.

Analizando por separado las lluvias de primavera (abril, mayo y junio) y de verano (julio, agosto y septiembre) se observa que el mayor descenso porcentual de la precipitación durante el intervalo 1598-1651 se debe más a la disminución que experimenta el trimestre estival $(-25 \%)$ que al primaveral $(-15 \%)$.

A partir de información documental referida a estas mismas décadas, también se han descrito momentos caracterizados por reducidas precipitaciones en otras zonas de España. Tal es el caso de Cataluña donde Barriendos"' señala la presencia de frecuentes y prolongadas sequías entre los años 1600-1620 y en las cuencas del Ebro y Duero donde $\mathrm{Saz}^{12}$ identifica secuencias de primaveras secas en estas mismas décadas, mientras que en las dos siguientes son los meses de verano los que soportan las mayores sequías en dichas cuencas. Por el contrario, Rodrigo et $a l^{13}$ no señalan condiciones secas destacables en Andalucía durante la primera mitad del siglo XVII, si bien ha de prevenirse que en este

11. BARriendos, M.: Climatologia histórica de Cataluña. Aproximación a sus caracteristicas generales (ss XV-XIX). Tesis Doctoral. Barcelona, 1994, págs 500.

12. SAZ-SÁNChEZ, M. A.: Temperaturas y precipitaciones en la mitad norte de España desde el siglo XV. Estudio dendroclimático. Zaragoza, 2003, págs 294.

13. Rodrigo, F., Esteban-Parra, M. J., Pozo VázQuez, D. y Castro-Diez, Y.: «A 500-year precipitation record in southern Spain», International Journal of Climatology, vol 19, 1999, págs 1233-1253. 
caso se trata de una valoración de los totales anuales de precipitación que perfectamente pueden enmascarar condiciones secas en determinados momentos el año.

\subsection{2.- El periodo lluvioso entre 1650 y 1670}

El periodo anterior termina con una fase moderadamente húmeda en la que los valores de precipitación vuelven a ser importantes. Corresponde a las décadas de los años cincuenta y sesenta del siglo XVII y debido en gran medida a una notable recuperación de la precipitación de primavera.

\begin{tabular}{|l|c|c|c|c|c|c|}
\hline & \multirow{2}{*}{$\begin{array}{l}\text { Intervalos } \\
\text { más secos }\end{array}$} & \multicolumn{2}{|c|}{ Anomalía } & Intervalos más & \multicolumn{2}{|c|}{ Anomalía } \\
\cline { 7 - 8 } & $\mathbf{m m}$ & $\%$ & lluviosos & mm & $\%$ \\
\hline & $1601-1610$ & $-28,6$ & $-33,7$ & $1715-1724$ & $+27,7$ & $+32,7$ \\
\hline & $1679-1687$ & $-26,6$ & $-31,4$ & $1829-1837$ & $+22,9$ & $+27,0$ \\
\hline & $1642-1651$ & $-23,0$ & $-27,1$ & $1564-1573$ & $+20,1$ & $+23,7$ \\
\hline & $1629-1638$ & $-16,7$ & $-19,7$ & $1883-1892$ & $+18,8$ & $+22,2$ \\
\hline & $1772-1781$ & $-16,7$ & $-19,7$ & $1790-1799$ & $+17,4$ & $+20,5$ \\
\hline años años & $1647-1651$ & $-39,6$ & $-46,7$ & $1833-1837$ & $+44,8$ & $+52,8$ \\
\hline & $1604-1608$ & $-37,0$ & $-43,6$ & $1719-1723$ & $+36,9$ & $+43,5$ \\
\hline & $1681-1685$ & $-31,0$ & $-36,6$ & $1569-1573$ & $+30,9$ & $+36,4$ \\
\hline & $1611-1615$ & $-27,6$ & $-32,5$ & $1794-1798$ & $+29,1$ & $+34,3$ \\
\hline & $1867-1871$ & $-27,5$ & $-32,4$ & $1860-1864$ & $+27,6$ & $+32,5$ \\
\hline 3 años & $1649-1651$ & $-49,2$ & $-58,0$ & $1834-1836$ & $+52,0$ & $+69,3$ \\
\hline & $1613-1615$ & $-45,2$ & $-53,3$ & $1722-1724$ & $+42,5$ & $+50,1$ \\
\hline & $1680-1682$ & $-39,9$ & $-47,1$ & $1811-1813$ & $+40,3$ & $+47,5$ \\
\hline & $1604-1606$ & $-39,1$ & $-46,1$ & $1569-1571$ & $+37,3$ & $+44,0$ \\
\hline & $1608-1610$ & $-37,3$ & $-44,0$ & $1554-1556$ & $+34,9$ & $+41,2$ \\
\hline años & 1608 & $-62,1$ & $-73,2$ & 1555 & $+64,1$ & $+75,6$ \\
\hline & 1605 & $-57,6$ & $-67,9$ & 1863 & $+63,1$ & $+74,4$ \\
\hline & 1615 & $-54,8$ & $-64,6$ & 1834 & $+58,5$ & $+69,0$ \\
\hline & 1649 & $-50,8$ & $-59,9$ & 1693 & $+58,4$ & $+68,9$ \\
\hline & 1557 & -49.9 & $-58,8$ & 1836 & $+56,1$ & $+66,2$ \\
\hline
\end{tabular}

Tabla 3.-- Periodos con mayor anormalidad positiva (periodos lluviosos) o negativa (sequias) calculados para distintos intervalos de tiempo.

\subsection{3.- El periodo seco de 1671 a 1687}

La segunda de las fases secas destacadas tiene lugar en los años setenta y ochenta del siglo XVII. Se corresponde con uno de los episodios climáticos más interesantes del último milenio, el Mínimo Maunder, caracterizado por un descenso de las temperaturas 
en Europa ${ }^{14}$, a la vez que por una notable inestabilidad interanual de las precipitaciones ${ }^{15}$.

En el sur de la provincia de Alicante, y considerando el periodo cálido en su conjunto, la precipitación entre 1671 y 1687 se reduce un $23,5 \%$ respecto de la media de los siglos analizados y sólo en tres ocasiones supera ese umbral. Frente a lo acontecido en la primera mitad del XVII, en esta ocasión el descenso de la precipitación es mayor durante las primaveras, que ven reducida su cuantía en un $28 \%$ frente a un $20 \%$ en verano. Rodrigo et $a l^{16}$ también señalan una disminución de la lluvia anual en Andalucía, al igual que $\mathrm{Saz}^{17}$ lo detecta en las primaveras y veranos de la mitad norte de España, lo cual permite concluir que se trató de un hecho bastante generalizado en el ámbito español. Por el contrario Barriendos ${ }^{18}$ no señala condiciones de sequía en Cataluña, aunque advierte de la elevada irregularidad interanual de la precipitación durante este periodo.

\subsection{4- Incremento de la precipitación entre 1688 y 1739}

A finales del siglo XVII y durante el primer tercio del XVIII se produce un aumento general y continuado de las precipitaciones en esta zona, aunque no de forma espectacular. Su incremento puede cuantificarse en sólo un $21 \%$ superior respecto de los $84,8 \mathrm{~mm}$ de media debido a la presencia de grupos de años con escasa pluviometría, como ocurrió con la mayoría de las primaveras entre 1706 y 1715. Algunos autores incluso destacan la presencia de sequías o lluvias torrenciales, que no por ser poco duraderas son menos importantes y en cualquier caso indicadoras de una elevada variabilidad climática. Tal es la fuerte sequía ocurrida en 1725 y los aguaceros del verano de 1728 detectados en la provincia de Valencia (19) que también dejaron sentir sus efectos en la zona alicantina como lo prueban los valores de precipitación reconstruidos durante dichos años.

Tras un largo periodo de características más o menos húmedas, las precipitaciones tienden a disminuir y los periodos secos empiezan a ser más frecuentes a partir de mediados del siglo XVIII en que las sequías se generalizan.

14. Luterbacher, J., Rickli, R., Xoplaki, E., Tinguely, C., Beck, C., Pfister, C. y Wanner, H.: «The late Maunder Minimum (1675-1715). A key period for studying decadal scale climatic change in Europe». Climatic Change, vol 49, 2001, págs 441-462.

15. Alcoforado, M. J., Nunes, M. F., García, J. C. y TABorda J. P. (2000). «Temperature and precipitation reconstruction in southern Portugal during the late Maunder Minimum (AD 1675-1715)». The Holocene, vol 10 (3), 2000, págs 333-340.

16. Rodrigo, F., Esteban-parra, M. J., Pozo Vázquez, D. y Castro-Díez, Y.: «A 500-year precipitation record in southern Spain». International Journal of Climatology, vol 19, 1999, págs 1233-1253.

17. SAZ-SÁncheZ, M. A.: Temperaturas y precipitaciones en la mitad norte de España desde el siglo XV. Estudio dendroclimático. Zaragoza, 2003, págs 294.

18. BARRIENDOS, M.: Climatología histórica de Cataluña. Aproximación a sus características generales (ss $X V-X L X)$. Tesis Doctoral. Barcelona, 1994, págs 500. 


\subsection{5.- Las décadas secas de los años sesenta y setenta del siglo XVIII}

La reducción de las lluvias a partir de la década de los sesenta de este siglo marca el comienzo de una fase seca que, de modo más o menos alternante, se prolonga hasta los años ochenta. Las sequías son muy frecuentes y la crisis pluviométrica se vuelve especialmente acusada en torno a los años setenta.

Analizado este periodo con más detalle se observa cómo entre 1764 y 1781 sólo dos años tienen una precipitación estival superior a la media, quedando en su conjunto un $30,4 \%$ por debajo de ese umbral. Este descenso de la precipitación se manifiesta tanto en primavera como en verano, como lo indican los similares decrementos de los valores de ambos periodos: un $29,9 \%$ y un $28,2 \%$, respectivamente.

Este periodo seco ha sido especialmente estudiado en Cataluña a través de la documentación escrita y donde lo identifican con el nombre de Pulsación Malda ${ }^{20}$. Otros autores indican asimismo la presencia de condiciones secas en la mitad norte de España en estos mismos años ${ }^{21}$, al igual que en Andalucía ${ }^{22}$, lo que permite identificar estos decenios como un periodo de sequías bastante generalizadas en gran parte de territorio español.

\subsection{6.- El discurrir del siglo XIX}

Finalizadas estas fases secas que afectan a gran parte de la segunda mitad del siglo XVII, se inicia una alternancia de periodos húmedos y secos caracterizados por una menor duración, aunque de intensidad bastante parecida. Efectivamente, durante el tramo final del siglo XVIII y todo el siglo XIX la precipitación del periodo cálido sigue mostrando oscilaciones de carácter seco y húmedo, pero su duración rara vez supera los 5-10 años, por supuesto intercalados de años con precipitaciones de signo contrario al que caracteriza el grupo de años agrupados. Como ejemplo de periodo seco cabe señalar la sequía que globalmente afectó a los años 1867-1879 y que durante el periodo cálido supuso un descenso del $32 \%$ de la precipitación.

Trabajos recientes realizados en esta zona, basados en el estudio de documentación escrita durante el siglo $\mathrm{XIX}^{23}$, permiten comparar los resultados obtenidos con estos dos tipos de fuentes. A modo de ejemplo destacaremos algunos de ellos. Tanto la dendroclimatología como la documentación escrita sobre rogativas «pro pluviam» coinciden en señalar el primer decenio de este siglo como un periodo seco bastante prolongado y que, según los resultados dendroclimáticos, la escasez de lluvias afectó

19. AlbEROLA, A.: La percepción de la catástrofe: sequia e inundaciones en tierras valencianas durante la primera mitad del siglo XVIII. Revista de Historia Moderna, vol 15, Alicante, 1996, 257-269.

20. BarRIEndos, M.: Climatologia histórica de Cataluña. Aproximación a sus características generales (ss XV-XIX). Tesis Doctoral. Barcelona, 1994, págs 500.

21. SAZ-SANCHEZ, M. A.: Temperaturas y precipitaciones en la mitad norte de España desde el siglo XV. Estudio dendroclimático. Zaragoza, 2003, págs 294.

22. Rodrigo, F., Esteban-Parra, MJ., Pozo Vazquez, D. y Castro-Diez, Y.: «A 500-year precipitation record in southern Spain». International Journat of Climatology, vol 19, 1999, págs 1233-1253.

23. ZAMORA, R. (2002): El final de la Pequeña Edad del Hielo en Alicante, Alicante, 2002, págs 194. 
tanto a las primaveras como a los veranos, aunque algo más a las primeras. Igual coincidencia muestran ambas fuentes de información con la sequía que afectó al segundo decenio, o la que se prolongó durante casi todo el cuarto decenio. Según los datos dendroclimáticos, ambas incidieron más en el trimestre primaveral. Las fases húmedas más destacables, corroborada también por ambas fuentes, afectaron a los años treinta y ochenta de este siglo XIX. Aquella fue una de las más intensas y ésta una de las más duraderas desde mediados del siglo XVIII, y ambas afectaron en mayor medida a las primaveras.

En estas alteraciones más o menos cíclicas habidas a mediados del siglo XIX, de menor duración que las de siglos anteriores, se suelen situar las últimas manifestaciones de la Pequeña Edad de Hielo (PEH) en España. En este sentido sus conclusiones coinciden con los resultados dendroclimáticos aquí presentados, que muestran cómo la variabilidad de la precipitación estival (expresada por el coeficiente de variación) aumenta hasta mediados del siglo XIX y que parece corresponder a algún tipo de pulsación climática. Pero, a la vez, estas reconstrucciones también indican que hasta finales del siglo XIX se produjeron pulsaciones secas y húmedas casi tan destacables como las habidas durante la primera mitad de este siglo.

Usando el concepto de variabilidad, entendida como la frecuencia de valores extremos (cálidos y fríos, secos y húmedos) obtenidos con reconstrucciones dendroclimáticas españolas, otros autores ${ }^{24}$ adelantan el final de dicho periodo frío (PEH) a mediados del siglo XVIII, momento en que las pulsaciones climáticas comienzan a disminuir tanto su intensidad como frecuencia.

Por tanto, las anomalías de mediados del siglo XIX quedan separadas de la gran variabilidad habida en los siglos XVI, XVII y mediados del XVIII (PEH) por un periodo bastante más estable que ocupa gran parte de la segunda mitad del siglo XVIII. Ello plantea muchas dudas respecto de si las oscilaciones del siglo XIX son parte del mismo proceso que dio lugar a la PEH y que marcarían el final de la misma o forman parte de otras anomalías climáticas, de menor intensidad pero más recurrentes en el tiempo, que nada tienen que ver con aquella.

Evidentemente, el clima es un sistema muy complejo y la reconstrucción de sus manifestaciones a pequeña escala no está exenta de dificultades, en especial cuando se trata de sequías, aguaceros y otros acontecimientos que pueden tener comportamientos espaciales muy distintos en cortas distancias. En este sentido, todas las fuentes de información realizan aportaciones imprescindibles y todas avanzan por un camino no finalizado.

\section{5.- CONCLUSIONES}

Los valores de precipitación reconstruidos en el sur de la provincia de Alicante (Elche) a partir de dendrocronologías han permitido conocer la evolución de las lluvias

24. MANRiQUE, E. y FERNÁNDEZ, A. (2000): «Extreme climatic events in dendroclimatic reconstructions from Spain». Climatic Change, vol. 44, 2000, 123-138. 
primaverales y estivales (denominadas «periodo cálido» cuando las agrupamos) desde mediados del siglo XVI hasta comienzos del siglo XX.

Su secuencia temporal muestra los constantes cambios que experimenta el clima a lo largo de los siglos, ya sea como pulsaciones más o menos amplias o variaciones interanuales muy cortas. En cualquier caso su mutabilidad es evidente.

Los periodos analizados los hemos denominado secos o húmedos en función del predominio de las sequías o las lluvias, sin que en unos y otros se excluya la presencia de años de características contrarias.

El análisis temporal de los valores reconstruidos permite destacar la disminución de las precipitaciones durante la primera mitad del siglo XVII, al que sigue una fase relativamente húmeda que se prolonga hasta los años 70 de este mismo siglo. Desde este momento y durante dos décadas vuelven a dominar los años secos que dan lugar a un periodo de fuerte variabilidad pluviométrica.

El siglo XVIII se inicia con una fase húmeda que ya arranca en el último decenio del siglo anterior y se prolonga hasta los años cuarenta. Tras unos años de indefinición coincidentes con la mitad del siglo, en los años sesenta se inicia un periodo de frecuentes sequías que persisten hasta comienzo de la década de los ochenta y caracterizado por una menor variabilidad pluviométrica.

Por último, el siglo XIX sigue mostrando esta alternancia de pulsaciones secas y húmedas, pero menos duraderas que las de siglos anteriores en que la $\mathrm{PEH}$ se manifestó en su plenitud y que deben interpretarse como una tendencia hacia una mayor normalidad climática.

\section{AGRADECIMIENTOS}

Parte de este trabajo se llevo a cabo durante el proyecto CLI96-1862 «Precipitaciones y temperaturas de la mitad septentrional de España a partir del siglo XVII $\gg$ financiado por la Comisión Interministerial de Ciencia y Tecnología CICYT. Actualmente se encuentra enmarcado dentro del Programa de grupos de investigación consolidados (grupo Clima, Cambio Global y Sistemas Naturales) financiado por el Gobierno de Aragón. 